\title{
Electromagnetic Field Correlators, Maxwell Stress Tensor, and the Casimir Effect for Parallel Walls
}

\author{
F. C. Santos, J. J. Passos Sobrinho, and A. C. Tort \\ Instituto de Física, Universidade Federal do Rio de Janeiro, \\ Cidade Universitária - Ilha do Fundão - Caixa Postal 68528, 21945-970 Rio de Janeiro RJ, Brazil
}

Received on 23 May, 2005

\begin{abstract}
We evaluate the quantum electromagnetic field correlators associated with the electromagnetic vacuum distorted by the presence of two plane parallel conducting walls and in the presence of a conducting wall parallel to a perfectly magnetically permeable one. Regularization is performed through the generalized zeta funtion technique. Results are applied to rederive the atractive and repulsive Casimir effect through Maxwell stress tensor. Surface divergences are shown to cancel out when stresses on both sides of the material surface are taken into account.
\end{abstract}

\section{INTRODUCTION}

According to Casimir [1], the (macroscopically) observable vacuum energy of a quantum field is the regularized difference between the zero point energies with and without the external conditions demanded by the particular physical situation at hand. In the case of the quantized electromagnetic field confined between two infinite parallel conducting walls separated by a distance $a$, Casimir's conception of the vacuum energy leads to a force per unit area between the walls given by

$$
\frac{F}{A}=-\frac{\pi \hbar c}{240 a^{4}} \text {. }
$$

Until 1997 only one experiment involving Casimir's original setup had been performed [2]. Lately, however, the experimental observation of this tiny force with metallic surfaces was significantly improved by a series of new experiments due to Lamoreaux, Mohideen and Roy, and Mohideen [3]. The concept of an observable vacuum energy can be extended to all quantum fields and several types of boundary conditions and/or applied external fields. An updated review of all these Casimir effects can be found in the monograph by Bordag, Mohideen, and Mostepananko [4], but see also, Mostepanenko and Trunov, and Plunien et al. [5].

The local approach to the electromagnetic Casimir effect was initiated by Brown and Maclay who calculated the renormalized stress energy tensor between two parallel perfectly conducting plates by means of Green functions techniques [6]. An interesting approach to the standard Casimir effect is the one due to Gonzales [7]. Analyzing the vacuum oscillations of the electromagnetic field confined by two parallel slabs separated by a gap of size $a$, Gonzales pointed out that the apparently non-objectionable definition of the vacuum energy given above could easily lead to conceptual errors and stressed the fact that in any Casimir interaction calculation, contributions from both sides of the material surfaces involved must be taken into account. This is so because the vacuum pressure always pushes the material surfaces involved, therefore, the repulsiveness or atractiveness of the Casimir force depends on the discontinuity of the relevant component of the quantized Maxwell stress tensor at the location of the surface.

The purpose of this paper is to pursue this line of reasoning by analyzing the stresses on parallel plane walls due to the vacuum distortions caused by the presence of these walls through the quantized version of the Maxwell stress tensor. Though the approach chosen here has many points in common with Ref. [7] cited above and Green functions techniques [8], it is a different alternative in the sense that it relies on objects known as correlators, which are regularized vacuum expectation values of products of the electromagnetic field components taken at the same point of space and for equal times. These correlators contain all the information we need about the local behavior of the vacuum expectation values of elements of Maxwell stress tensor, in particular, their behavior near both sides of the material surface in question, a feature crucial to the obtention of the correct result. The local behavior of the Maxwell tensor, or of the relativistic symmetrical stress-energy tensor, is extremely important because as shown by, for example, Deutsch and Candelas [9] with the help of Green functions techniques, as we approach the boundaries we find strong divergencies that cannot be simply removed by usual renormalization procedures. Besides reviewing the obtention of the electromagnetic Casimir for the standard case of two perfectly conducting parallel walls, we will also consider a pair of parallel walls, one of them perfectly permeable. This setup was first proposed by Boyer [10] who analyzed them from the viewpoint of random electrodymanics and it is the simplest example of a repulsive Casimir force. For both cases, the conducting plate and Boyer's setup we also construct the symmetrical stress tensor. We will employ Gaussian units and set $c=\hbar=1$.

\section{MAXWELL STRESS TENSOR AND THE ELECTROMAGNETIC FIELD CORRELATORS}

Our aim in this section is to obtain an expression for the quantum version of the electromagnetic force per unit area that acts on plane material wall. Material wall here means a perfectly conducting square $\operatorname{surface}(\varepsilon \rightarrow \infty)$ or a perfectly permeable one $(\mu \rightarrow \infty)$ whose linear dimension $L$ is much larger than others relevant dimensions envolved such as the distance between two of those walls. The physical interaction between any of the two types of walls considered here and the vacuum electromagnetic field is mimicked by the imposition of appropriate boundary conditions on the electromagnetic field 
on the location of the walls. The Cartesian components of the Maxwell stress tensor (in Gaussian units) are given by [11]

$$
T_{i j}=\frac{1}{4 \pi}\left(E_{i} E_{j}-\frac{1}{2} \delta_{i j} \mathbf{E}^{2}+B_{i} B_{j}-\frac{1}{2} \delta_{i j} \mathbf{B}^{2}\right)
$$

where $i, j=x, y, z$. Suppose that the material surface is placed perpendicularly to the $O Z$ axis. Upon quantizing the electromagnetic field we can write the quantum version of (2). For instance,

$$
\left\langle\hat{T}_{z z}\right\rangle_{0}=\frac{1}{8 \pi}\left[\left\langle\hat{E}_{z}^{2}\right\rangle_{0}-\left\langle\hat{E}_{\|}^{2}\right\rangle_{0}+\left\langle\hat{B}_{z}^{2}\right\rangle_{0}-\left\langle\hat{B}_{\|}^{2}\right\rangle_{0}\right],
$$

where $\hat{E}_{\|}^{2}=\hat{E}_{x}^{2}+\hat{E}_{y}^{2}$ and $\hat{B}_{\|}^{2}=\hat{B}_{x}^{2}+\hat{B}_{y}^{2} ;\langle\hat{O}\rangle_{0} \equiv\langle 0|\hat{O}| 0\rangle$ denotes a vacuum expectation value. The other Cartesian components of this tensor can be obtained in an analogous way. The quantum macroscopic force $\langle\hat{\mathbf{F}}\rangle_{0}$ on the wall can be evaluated by integrating the quantum version of the classical result [11]

$$
\mathbf{F}=\int_{\partial \mathcal{R}} \tilde{\mathbf{T}} \cdot \hat{\mathbf{n}} d a
$$

were $\hat{\mathbf{n}}$ is the outward normal at the boundary $\partial \mathcal{R}$ and $\mathcal{R}$ is any region containing all or part of the wall. Classically, (4) can be obtained by integrating the Lorentz force per unit volume acting on charge and current distributions and eliminating the sources in favor of the fields. From a quantum point of view, we see that the problem of evaluating the pressure the material surface due to the distorted zero point oscillations of the electromagnetic field is reduced to the evaluation of the vacuum expectation value of the quantum operators $\hat{E}_{i}(\mathbf{r}, t) \hat{E}_{j}(\mathbf{r}, t)$, $\hat{B}_{i}(\mathbf{r}, t) \hat{B}_{j}(\mathbf{r}, t)$, and $\hat{E}_{i}(\mathbf{r}, t) \hat{B}_{j}(\mathbf{r}, t)$. The evaluation of these correlators depends on the specific choice of the boundary conditions. A regularization recipe will also be necessary, for these objects are mathematically ill-defined. For the setup envolving conducting plates these correlators were evaluated by Lütken and Ravndal [16], see also [12]. They can be also obtained from the coincidence limit of the photon propagator between conducting plates evaluated by Bordag et al [17]. In the next section we will evaluate and regularize these correlators by means of analitycal continuation techniques [13] similar, though not equal, to the ones employed by Lütken e Ravndal [16]. We will also show how to obtain the corresponding results for another unusual but intersting setup [13].

\section{CORRELATORS FOR CASIMIR'S SETUP}

Consider an experimental setup consisting in two infinite perfectly conducting parallel plates $(\varepsilon \rightarrow \infty)$ kept at a fixed distance $a$ from each other. We will choose the coordinates axis in such a way that the $O Z$ direction is perpendicular to the plates. One of the plates will be placed at $z=0$ and the other one at $z=a$. The field must satisfy the following boundary conditions on the plates: the tangential components $E_{x}$ e $E_{y}$ of the electric field and the normal component $B_{z}$ of the magnetic field must be zero on the plates. Since there are no real charges or currents it will be convenient to work in the Coulomb gauge in which $\nabla \cdot \mathbf{A}(\mathbf{r}, t)=0$, and $\Phi=0$, thus $\mathbf{E}(\mathbf{r}, t)=-\partial \mathbf{A}(\mathbf{r}, t) / \partial t$ and $\mathbf{B}(\mathbf{r}, t)=\nabla \times \mathbf{A}(\mathbf{r}, \mathbf{t})$. These physical boundary conditions combined with the choice of gauge allow us to rewrite the boundary conditions in terms of the components of the vector potential $\mathbf{A}(\mathbf{r}, t)$ in the following way: at $z=0$ we will have,

$$
\begin{gathered}
A_{x}(x, y, 0, t)=0 ; \quad A_{y}(x, y, 0, t)=0 ; \\
\frac{\partial}{\partial z} A_{z}(x, y, 0, t)=0,
\end{gathered}
$$

and at $z=a$,

$$
\begin{gathered}
A_{x}(x, y, a, t)=0 ; \quad A_{y}(x, y, a, t)=0 ; \\
\frac{\partial}{\partial z} A_{z}(x, y, a, t)=0 .
\end{gathered}
$$

The vector potential operator $\hat{\mathbf{A}}(\mathbf{r}, t)$ that satisfies the wave equation, the Coulmb gauge and the boundary conditions can be written as

$$
\begin{aligned}
\hat{\mathbf{A}}(\mathbf{r}, t) & =\frac{1}{\pi}\left(\frac{\pi}{a}\right)^{\frac{1}{2}} \sum_{n=0}^{\infty} \int \frac{d^{2} \kappa}{\sqrt{\omega}}\left\{\hat{a}^{(1)}(\kappa, n) \hat{\kappa} \times \hat{\mathbf{z}} \sin \left(\frac{n \pi z}{a}\right)\right. \\
& \left.+\hat{a}^{(2)}(\kappa, n)\left[\hat{\kappa} \frac{i n}{\omega a} \sin \left(\frac{n \pi z}{a}\right)-\hat{\mathbf{z}} \frac{\kappa}{\omega} \cos \left(\frac{n \pi z}{a}\right)\right]\right\} e^{i(\kappa \cdot \rho-\omega t)}+\text { h.c },
\end{aligned}
$$

where $\kappa=\left(k_{x}, k_{y}\right)$ and $\rho$ is the position arrow on the $\chi \mathcal{Y}$ plane. The normal frquencies are given by

$$
\omega=\omega(\kappa, n)=\sqrt{\kappa^{2}+n^{2} \frac{\pi^{2}}{a^{2}}}
$$

with $k_{x}, k_{y} \in \mathbb{R}$ e $n \in \mathbb{N}-1$. The symbol $\sum$ " indicates that the term corresponding to $n=0$ for normaliztion reasons must be multiplied by $1 / 2$. The Fourier coefficients $\hat{a}^{(\lambda)}(\kappa, n)$ where $\lambda=1,2$ is the polarization index, are operators in the photon 
ocupation number space and satisfy

$$
\left[\hat{a}^{(\lambda)}(\kappa, n), \hat{a}^{\dagger\left(\lambda^{\prime}\right)}\left(\kappa^{\prime}, n^{\prime}\right)\right]=\delta_{\lambda \lambda^{\prime}} \delta_{n n^{\prime}} \delta\left(\kappa-\kappa^{\prime}\right)
$$

It is convenient to write the vector potential in the general form

$$
\hat{\mathbf{A}}(\mathbf{r}, t)=\sum_{n=0}^{\infty} \int d^{2} \kappa \sum_{\lambda=1}^{2} \hat{a}^{(\lambda)}(\kappa, n) \mathbf{A}_{\kappa n}^{(\lambda)}(\mathbf{r}) e^{-i \omega(\kappa, n) t}+\text { h.c },
$$

where $\mathbf{A}_{\kappa n}^{(\lambda)}(\mathbf{r})$ are the modal functions. The modal functions for each polarization state must obey Helmholtz equation and the boundary conditions given above. In our case the modal functions are

$$
\mathbf{A}_{\kappa n}^{(1)}(\mathbf{r})=\frac{1}{\pi}\left(\frac{\pi}{a}\right)^{\frac{1}{2}} \frac{1}{\sqrt{\omega}} \sin \left(\frac{n \pi z}{a}\right) e^{-i \kappa \cdot \rho} \hat{\kappa} \times \hat{\mathbf{z}},
$$

and

$$
\mathbf{A}_{\kappa n}^{(2)}(\mathbf{r})=\frac{1}{\pi}\left(\frac{\pi}{a}\right)^{\frac{1}{2}} \frac{1}{\sqrt{\omega}}\left[\hat{\kappa} \frac{i n \pi}{a \omega} \sin \left(\frac{n \pi z}{a}\right)-\hat{\mathbf{z}} \frac{\kappa}{\omega} \cos \left(\frac{n \pi z}{a}\right)\right] e^{-i \kappa \cdot \rho} .
$$

The next step is to evaluate the electric field operator $\hat{\mathbf{E}}(\mathbf{r}, t)$. Recalling that $\hat{a}^{(\lambda)}(\kappa, n)|0\rangle=0$, we first write the correlators $\left.<\hat{E}_{i}(\mathbf{r}, t) \hat{E}_{j}(\mathbf{r}, t)\right\rangle_{0}$ in the general form

$$
\left.<\hat{E}_{i}(\mathbf{r}, t) \hat{E}_{j}(\mathbf{r}, t)\right\rangle_{0}=\sum_{\alpha} E_{i \alpha}(\mathbf{r}) E_{j \alpha}^{*}(\mathbf{r})
$$

where we have introduced the modal functions $E_{i \alpha}(\mathbf{r})$ for the electric field. In our case (11) and (12) yield

$$
\mathbf{E}_{i \kappa n}^{(1)}(\mathbf{r})=\frac{i}{\pi}\left(\frac{\omega(\kappa, n) \pi}{a}\right)^{\frac{1}{2}} \sin \left(\frac{n \pi z}{a}\right) e^{-i \kappa \cdot \rho}(\hat{\kappa} \times \hat{\mathbf{z}})_{i},
$$

and,

$$
\mathbf{E}_{i \kappa n}^{(2)}(\mathbf{r})=\frac{i}{\pi}\left(\frac{\omega(\kappa, n) \pi}{a}\right)^{\frac{1}{2}}\left[\kappa_{i} \frac{i n \pi}{a \omega(\kappa, n)} \sin \left(\frac{n \pi z}{a}\right)-\hat{\mathbf{z}}_{i} \frac{\kappa}{\omega(\kappa, n)} \cos \left(\frac{n \pi z}{a}\right)\right] e^{-i \kappa \cdot \rho},
$$

respectively. Taking (14) and (15) into (13), we write $\hat{\kappa}_{i}=\cos \phi \delta_{i x}+\sin \phi \delta_{i y}, \hat{\mathbf{z}}_{i}=\delta_{i z} \mathrm{e}(\hat{\mathbf{z}} \times \hat{\mathbf{\kappa}})_{i}=\sin \phi \delta_{i x}-\cos \phi \delta_{i y}$, where $\phi$ is the azimuthal angle on the $X \mathcal{Y}$ plane and we have performed all angular integrals. In this way we end up with

$$
\begin{aligned}
& \left\langle\hat{E}_{i}(\mathbf{r}, t) \hat{E}_{j}(\mathbf{r}, t)\right\rangle_{0}=\left(\frac{2}{\pi}\right)\left(\frac{\pi}{a}\right) \frac{\delta_{i j}^{\|}}{2} \sum_{n=0}^{\infty} \sin ^{2}\left(\frac{n \pi z}{a}\right) \int_{0}^{\infty} d \kappa \kappa \omega(\kappa, n) \\
+ & \left(\frac{2}{\pi}\right)\left(\frac{\pi}{a}\right)\left(\frac{\pi}{a}\right)^{2} \frac{\delta_{i j}^{\|}}{2} \sum_{n=0}^{\infty \prime \prime} n^{2} \sin ^{2}\left(\frac{n \pi z}{a}\right) \int_{0}^{\infty} d \kappa \kappa \omega^{-1}(\kappa, n) \\
+ & \left(\frac{2}{\pi}\right)\left(\frac{\pi}{a}\right) \delta_{i j}^{\perp} \sum_{n=0}^{\infty} \cos ^{2}\left(\frac{n \pi z}{a}\right) \int_{0}^{\infty} d \kappa \kappa^{3} \omega^{-1}(\kappa, n),
\end{aligned}
$$

where $\delta_{i j}^{\|}:=\delta_{i x} \delta_{j x}+\delta_{i y} \delta_{j y}$ e $\delta_{i j}^{\perp}:=\delta_{i z} \delta_{j z}$. Equation (16) is a formal expression for the correlator $\left\langle E_{i}(\mathbf{r}, t) E_{j}(\mathbf{r}, t)\right\rangle_{0}$, since it is mathematically ill-defined unless a regularization recipe is prescribed. We will regularize the integrals in (16) with the help of analytical continuation methods.

Consider, for instance, the first integral on the r.h.s. of (16) and let us rewrite it as follows

$$
\int_{0}^{\infty} d \kappa \kappa\left(\kappa^{2}+\frac{n^{2} \pi^{2}}{a^{2}}\right)^{1 / 2} \rightarrow \int_{0}^{\infty} d \kappa \kappa\left(\kappa^{2}+\frac{n^{2} \pi^{2}}{a^{2}}\right)^{1 / 2-s} .
$$

The first term in (16) can be rewritten as

$$
T_{1}=\left(\frac{2}{\pi}\right)\left(\frac{\pi}{a}\right) \frac{\delta_{i j}^{\|}}{2} \sum_{n=0}^{\infty ”} \sin ^{2}\left(\frac{n \pi z}{a}\right) \int_{0}^{\infty} d \kappa \kappa\left(\kappa^{2}+\frac{n^{2} \pi^{2}}{a^{2}}\right)^{\frac{1}{2}-s}
$$


the second as

$$
T_{2}=\left(\frac{2}{\pi}\right)\left(\frac{\pi}{a}\right)\left(\frac{\pi}{a}\right)^{2} \frac{\delta_{i j}^{\|}}{2} \sum_{n=0}^{\infty} n^{2} \sin ^{2}\left(\frac{n \pi z}{a}\right) \int_{0}^{\infty} d \kappa \kappa\left(\kappa^{2}+\frac{n^{2} \pi^{2}}{a^{2}}\right)^{-\frac{1}{2}-s}
$$

and the third one as

$$
T_{3}=\left(\frac{2}{\pi}\right)\left(\frac{\pi}{a}\right) \delta_{i j}^{\perp} \sum_{n=0}^{\infty} \cos ^{2}\left(\frac{n \pi z}{a}\right) \int_{0}^{\infty} d \kappa \kappa^{3}\left(\kappa^{2}+\frac{n^{2} \pi^{2}}{a^{2}}\right)^{-\frac{1}{2}-s}
$$

Let us assume that $\mathfrak{R} s$ is large enough to give precise mathematical meaning to these integrals. After evaluating them and make use of the analytical continuation of the results we will take the limit $s \rightarrow 0$. Let us see, for instance, what happens with $T_{1}$. Making use of the following representation of Euler beta function [19]

$$
\int_{0}^{\infty} d x x^{\mu-1}\left(x^{2}+c^{2}\right)^{v-1}=\frac{B}{2}\left(\frac{\mu}{2}, 1-v-\frac{\mu}{2}\right) c^{\mu+2 v-2},
$$

where $B(x, y)=\Gamma(x) \Gamma(y) / \Gamma(x+y)$, and that holds for $\Re\left(v+\frac{\mu}{2}\right)<1$ and $\Re \mu>0$, we obtain

$$
\int_{0}^{\infty} d \kappa \kappa\left(\kappa^{2}+\frac{n^{2} \pi^{2}}{a^{2}}\right)^{1 / 2-s}=\frac{1}{2}\left(\frac{n \pi}{a}\right)^{3-2 s} \frac{\Gamma(s-3 / 2)}{\Gamma(s-1 / 2)}=\frac{1}{(2 s-3)}\left(\frac{n \pi}{a}\right)^{3-2 s} .
$$

Taking this result into $T_{1}$ we obtain

$$
T_{1}=\left(\frac{1}{2 s-3}\right)\left(\frac{\pi}{a}\right)^{3-2 s} \frac{\delta_{i j}^{\|}}{2 a}\left[\zeta_{R}(2 s-3)-\sum_{n=0}^{\infty} n^{3-2 s} \cos \left(\frac{2 n \pi z}{a}\right)\right],
$$

where $\zeta_{R}(z)$ is the well-known Riemann zeta function. Taking the limit $s \rightarrow 0$, we have

$$
T_{1}=-\frac{1}{3 \pi}\left(\frac{\pi}{a}\right)^{4} \frac{\delta_{i j}^{\|}}{2}\left[\frac{1}{120}+\frac{1}{8} \sum_{n=1}^{\infty} \frac{d^{3}}{d \xi^{3}} \sin (2 n \xi)\right],
$$

where we made use of the fact that $\zeta_{R}(-3)=1 / 120$, defined $\xi:=\pi z / a$, and wrote

$$
n^{3} \cos (2 n \xi)=-\frac{1}{8} \times \frac{d^{3}}{d \xi^{3}} \sin (2 n \xi)
$$

The sum on the R.H.S. of (23) can be regularized in many ways. A quick though non-rigorous way is to write

$$
T_{1}=-\frac{1}{3 \pi}\left(\frac{\pi}{a}\right)^{4} \frac{\delta_{i j}^{\|}}{2}\left[\frac{1}{120}+\frac{1}{8} \frac{d^{3}}{d \xi^{3}} \sum_{n=1}^{\infty} \sin (2 n \xi)\right],
$$

and express the summand in terms of exponential functions of imaginary argument thereby transforming each one of the sums into the euclidean space. In this way we obtain

$$
\begin{aligned}
\sum_{n=1}^{\infty} \sin (2 n \xi) & =\frac{1}{2 i}\left(\sum_{n=1}^{\infty} \exp (i 2 n \xi)-\sum_{n=1}^{\infty} \exp (-i 2 n \xi)\right) \\
& =\frac{1}{2 i}\left(\sum_{n=1}^{\infty} \exp \left(2 n \xi_{E}\right)-\sum_{n=1}^{\infty} \exp \left(-2 n \xi_{E}^{\prime}\right)\right)
\end{aligned}
$$

where we have made the substitution $i \xi \rightarrow-\xi_{E}$ in the first sum and $i \xi \rightarrow \xi_{E}^{\prime}$ in the second. Each one of the sums above can be easily performed and the result is

$$
\sum_{n=1}^{\infty} \sin (2 n \xi)=\frac{1}{2} \cot (\xi)
$$


It follows that

$$
T_{1}=-\frac{1}{3 \pi}\left(\frac{\pi}{a}\right)^{4} \frac{\delta_{i j}^{\|}}{2}\left[\frac{1}{120}+\frac{1}{8} \frac{d^{3}}{d \xi^{3}} \frac{1}{2} \cot (\xi)\right] .
$$

Treating the two other terms in (16) in a similar manner we obtain

$$
T_{2}=-\frac{1}{\pi}\left(\frac{\pi}{a}\right)^{4} \frac{\delta_{i j}^{\|}}{2}\left[\frac{1}{120}+\frac{1}{8} \frac{d^{3}}{d \xi^{3}} \frac{1}{2} \cot (\xi)\right],
$$

and

$$
T_{3}=\frac{4}{3 \pi}\left(\frac{\pi}{a}\right)^{4} \frac{\delta_{i j}^{\perp}}{2}\left[\frac{1}{120}-\frac{1}{8} \frac{d^{3}}{d \xi^{3}} \frac{1}{2} \cot (\xi)\right] .
$$

Notice that some care must be taken when we aply this procedure to the third term. This is so because the term corresponding to $n=0$ in $T_{3}$ is not zero. In fact its contribution is:

$$
T_{3}(n=0)=\left(\frac{2}{\pi}\right)\left(\frac{\pi}{a}\right) \delta_{i j}^{\perp} \int_{0}^{\infty} d \kappa \kappa^{-2-2 s},
$$

which diverges when the regularization is removed. However this term is non-physical and can be safely ignored. Finally, collecting all partial results we have

$$
\begin{aligned}
\left\langle E_{i}(\mathbf{r}, t) E_{j}(\mathbf{r}, t)\right\rangle_{0} & =T_{1}+T_{2}+T_{3} \\
& =\left(\frac{\pi}{a}\right)^{4} \frac{2}{3 \pi}\left[\left(-\delta^{\|}+\delta^{\perp}\right)_{i j} \frac{1}{120}+\delta_{i j} F(\xi)\right] .
\end{aligned}
$$

The function $F(\xi)$ is defined by

$$
F(\xi):=-\frac{1}{8} \frac{d^{3}}{d \xi^{3}} \frac{1}{2} \cot (\xi)
$$

and its expansion about $\xi=0$ is given by

$$
F(\xi) \approx \frac{3}{8} \xi^{-4}+\frac{1}{120}+O\left(\xi^{2}\right)
$$

Near $\xi=\pi$ (which corresponds to $z=a$ ) we make the replacement $\xi \rightarrow \xi-\pi$. Notice that due to the behavior of $F(\xi)$ near $\xi=0, a$, strong divergences predominate in the behavior of the correlators near the plates.

By applying the exactly the same procedure we obtain the magnetic field correlators

$$
\left\langle B_{i}(\mathbf{r}, t) B_{j}(\mathbf{r}, t)\right\rangle_{0}=\left(\frac{\pi}{a}\right)^{4} \frac{2}{3 \pi}\left[\left(-\delta^{\|}+\delta^{\perp}\right)_{i j} \frac{1}{120}-\delta_{i j} F(\xi)\right] .
$$

A direct evaluation also shows that the correlators $<$ $\left.E_{i}(\mathbf{r}, t) B_{j}(\mathbf{r}, t)\right\rangle_{0}$ are zero.

\section{CORRELATORS FOR BOYER'S SETUP}

The other setup we are interested in is that one in which a perfectly conducting plate is placed at $z=0$ and perfectly permeable plate is placed at $z=a$. This setup was analyzed for the first time by Boyer in the contetxt of stochastic electrody- namic [10] and it is the simplest case of a repulsive Casimir effect that can be found in the literature. The boundary conditions now are: $(a)$ the tangential components $E_{x}$ and $E_{y}$ of the electric field as well as the normal component $B_{z}$ of the magnetic field must vanish on the surface of the plate at $z=0 ;(b)$ the tangential components of $B_{x}$ e $B_{y}$ of the magnetic field as well as normal conponent $E_{z}$ of the electric field must vanish on the surface of the plate at $z=a$. These boundary conditions translated in terms of the components of the vector potential read

$$
A_{x}(x, y, 0, t)=0 ; \quad A_{y}(x, y, 0, t)=0 ; \quad \frac{\partial}{\partial z} A_{z}(x, y, 0, t)=0,
$$


at $z=0$, and at $z=a$

$$
\frac{\partial}{\partial z} A_{x}(x, y, a, t)=0 ; \quad \frac{\partial}{\partial z} A_{y}(x, y, a, t)=0 ; \quad A_{z}(x, y, a, t)=0 .
$$

The appropriate vector potential operator $\hat{\mathbf{A}}(\mathbf{r}, t)$ is given by [13]

$$
\begin{aligned}
\hat{\mathbf{A}}(\mathbf{r}, t)= & \frac{1}{\pi}\left(\frac{\pi}{a}\right)^{\frac{1}{2}} \sum_{n=0}^{\infty} \int \frac{d^{2} \kappa}{\sqrt{\omega}}\left\{\hat{a}^{(1)}(\kappa, n) \hat{\kappa} \times \hat{\mathbf{z}} \sin \left[\left(n+\frac{1}{2}\right) \frac{\pi z}{a}\right]\right. \\
+ & \left.\hat{a}^{(2)}(\kappa, n)\left[\hat{\kappa} \frac{i\left(n+\frac{1}{2}\right)}{\omega a} \sin \left[\left(n+\frac{1}{2}\right) \frac{\pi z}{a}\right]-\hat{\mathbf{z}} \frac{\kappa}{\omega} \cos \left[\left(n+\frac{1}{2}\right) \frac{\pi z}{a}\right]\right]\right\} \\
& \times e^{i(\kappa \cdot \rho-\omega t)}+\text { h.c. },
\end{aligned}
$$

where as before $\kappa=\left(k_{x}, k_{y}\right)$ and $\rho$ is the position vector on the $\mathcal{X Y}$ plane. The normal frequencies are given

$$
\omega(\kappa, n)=\sqrt{\kappa^{2}+\left(n+\frac{1}{2}\right)^{2} \frac{\pi^{2}}{a^{2}}}
$$

with $k_{x}, k_{y} \in \mathbb{R}$ and $n \in \mathbb{N}-1$. Notice that contrary to the case of two conducting plates normalization does not require the we multiply the term corresponding to $n=0$ by $1 / 2$. The electric and magnetic field correlators for Boyer's setup can be evaluated with the same technique employed before [13]. In fact, it is not hard to convince ourselves that it is sufficient to perform the substitution $n \rightarrow n+1 / 2$ and follow the same steps as before to obtain

$$
\left\langle E_{i}(\mathbf{r}, t) E_{j}(\mathbf{r}, t)\right\rangle_{0}=T_{1}+T_{2}+T_{3}
$$

where, for example

$$
T_{1}=\frac{\Gamma\left(s-\frac{3}{2}\right)}{\Gamma\left(s-\frac{1}{2}\right)}\left(\frac{\pi}{a}\right)^{3-2 s} \frac{\delta_{i j}^{\|}}{2 a}\left\{\sum_{n=0}^{\infty}\left(n+\frac{1}{2}\right)^{3-2 s} \frac{1}{2}\left[1-\cos \left(2(n+1 / 2) \frac{\pi z}{a}\right)\right]\right\}
$$

The terms $T_{2}$ e $T_{1}$ show a similar structure. The main difference with respect to the two conducting plate case is that now we have to deal with the Hurwitz zeta function $\zeta_{H}(z, q)$ which has a series representation given by [19]

$$
\zeta_{H}(z, q)=\sum_{n=0}^{\infty} \frac{1}{(n+q)^{z}}
$$

with $\Re z>1$, and $q \neq 0,-1,-2, \ldots$. In our case we must set

$$
\zeta_{H}\left(2 s-3, \frac{1}{2}\right)=\sum_{n=0}^{\infty}\left(n+\frac{1}{2}\right)^{3-2 s} .
$$

It follows that in the limit $s \rightarrow 0$ we have

$$
T_{1}=-\left(\frac{\pi}{a}\right)^{4} \frac{1}{3 \pi} \frac{\delta_{i j}^{\|}}{2}\left\{\zeta_{H}\left(-3, \frac{1}{2}\right)+\sum_{n=0}^{\infty}\left(n+\frac{1}{2}\right)^{3} \cos \left(2\left(n+\frac{1}{2}\right) \frac{\pi z}{a}\right)\right\} .
$$

In the same way we obtain for $T_{2}$ e $T_{1}$ the results

$$
T_{2}=-\left(\frac{\pi}{a}\right)^{4} \frac{1}{\pi} \frac{\delta_{i j}^{\|}}{2}\left\{\zeta_{H}\left(-3, \frac{1}{2}\right)-\sum_{n=0}^{\infty}\left(n+\frac{1}{2}\right)^{3} \cos \left(2\left(n+\frac{1}{2}\right) \frac{\pi z}{a}\right)\right\}
$$

and

$$
T_{3}=\left(\frac{\pi}{a}\right)^{4} \frac{2}{3 \pi} \frac{\delta_{i j}^{\perp}}{2}\left\{\zeta_{H}\left(-3, \frac{1}{2}\right)+\sum_{n=0}^{\infty}\left(n+\frac{1}{2}\right)^{3} \cos \left(2\left(n+\frac{1}{2}\right) \frac{\pi z}{a}\right)\right\} .
$$


Notice that this time we do not have the divergent contribution corresponding to $n=0$ as in the case of the conducting plates. Adding the three terms we have

$$
\begin{aligned}
\left\langle\hat{E}_{i}(\mathbf{r}, t) \hat{E}_{j}(\mathbf{r}, t)\right\rangle_{0}= & \left(\frac{\pi}{a}\right)^{4} \frac{2}{3 \pi}\left\{\left(-\delta_{i j}^{\|}+\delta_{i j}^{\perp}\right) \zeta_{H}\left(-3, \frac{1}{2}\right)\right. \\
& \left.+\sum_{n=0}^{\infty}\left(n+\frac{1}{2}\right)^{3} \cos \left(2\left(n+\frac{1}{2}\right) \frac{\pi z}{a}\right)\right\} .
\end{aligned}
$$

The numerical value $\zeta_{H}\left(-3, \frac{1}{2}\right)$ can be obtained from [19]

$$
\zeta_{H}(-n, q)=-\frac{B_{n+1}(q)}{n+1}
$$

where $n \in \mathbf{N}$ and $B_{n+1}(q)$ is a Bernoulli polynomial defined by [19]

$$
B_{n}(x)=\sum_{p=0}^{n} \frac{n !}{p !(n-p) !} B_{p} x^{n-k}
$$

where $B_{p}$ is a Bernoulli number. The relevant polynomial here is:

$$
B_{4}(x)=x^{4}-2 x^{3}+x^{2}-\frac{1}{30}
$$

With $B_{4}(1 / 2)=(7 / 8) \times(1 / 30)$, it follows that $\zeta_{H}\left(-3, \frac{1}{2}\right)=$ $-(7 / 8)(1 / 120)$. The sum can be regularized with the same technique employed before. In fact, we can define the function $G(\xi)$ by

$$
\begin{aligned}
G(\xi) & :=\sum_{n=0}^{\infty}\left(n+\frac{1}{2}\right)^{3} \cos \left[2\left(n+\frac{1}{2}\right) \xi\right] \\
& =\frac{1}{8} \sum_{n=0}^{\infty}(2 n+1)^{3} \cos [(2 n+1) \xi],
\end{aligned}
$$

where as before $\xi:=z \pi / a$. We can write

$$
(2 n+1)^{3} \cos [(2 n+1) \xi]=-\frac{d^{3}}{d \xi^{3}} \sin [(2 n+1) \xi]
$$

and formally we have

$$
G(\xi)=-\frac{1}{8} \frac{d^{3}}{d \xi^{3}} \sum_{n=0}^{\infty} \sin [(2 n+1) \xi]
$$

Writing $\sin [(2 n+1) \xi]$ in terms of exponentials of imaginary argument and passing to the euclidean space we obtain after some simple manipulations

$$
G(\xi)=-\frac{1}{8} \frac{d^{3}}{d \xi^{3}} \frac{1}{2 \sin (\xi)}
$$

Collecting all partial results we finally obtain for $\left\langle\hat{E}_{i}(\mathbf{r}, t) \hat{E}_{j}(\mathbf{r}, t)\right\rangle_{0}$ the result

$$
\left\langle\hat{E}_{i}(\mathbf{r}, t) \hat{E}_{j}(\mathbf{r}, t)\right\rangle_{0}=\left(\frac{\pi}{a}\right)^{4} \frac{2}{3 \pi}\left[\left(-\frac{7}{8}\right) \frac{\left(-\delta^{\|}+\delta^{\perp}\right)_{i j}}{120}+\delta_{i j} G(\xi)\right] .
$$

Proceeding in the same way in the evaluation of $\left\langle\hat{B}_{i}(\mathbf{r}, t) \hat{B}_{j}(\mathbf{r}, t)\right\rangle_{0}$ we obtain

$$
\left\langle\hat{B}_{i}(\mathbf{r}, t) \hat{B}_{j}(\mathbf{r}, t)\right\rangle_{0}=\left(\frac{\pi}{a}\right)^{4} \frac{2}{3 \pi}\left[\left(-\frac{7}{8}\right) \frac{\left(-\delta^{\|}+\delta^{\perp}\right)_{i j}}{120}-\delta_{i j} G(\xi)\right] .
$$

Observe that near $\xi=0$ the function $G(\xi)$ behaves as

$$
G(\xi)=\frac{3}{8} \xi^{-4}-\frac{7}{8} \frac{1}{120}+O\left(\xi^{2}\right)
$$

But near $\xi=\pi$ its behavior is slightly different

$$
G(\xi)=-\frac{3}{8}(\xi-\pi)^{-4}+\frac{7}{8} \frac{1}{120}+O\left[(\xi-\pi)^{2}\right] .
$$

Again, a direct calculation shows that $\left\langle\hat{E}_{i}(\mathbf{r}, t) \hat{B}_{j}(\mathbf{r}, t)\right\rangle_{0}=0$ for this case. 


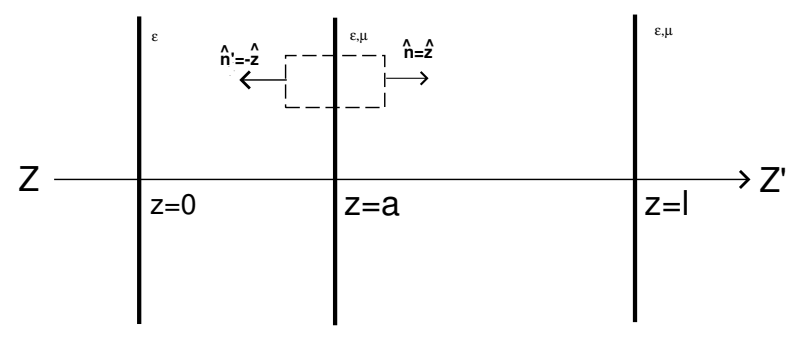

FIG. 1: Three-plate setup for the obtention of the Casimir force per unit area. The plate at $z=\ell$ is auxiliary.
As before the divergent behavior of the correlators near the plates we are interested in is an effect of the distortions of the electromagnetic oscillations with respect to a situation where the plates are not present. This fact has received the attention of several authors, see for example $[9,16]$.

\section{THE CASIMIR EFFECT FOR CONDUCTING PLATES}

In order to apply the above results to Casimir's original setup we consider for convenience three parallel perfectly conducting plates perpendicular to the $O Z$ axis at $z=0, z=a$ and $z=\ell$

The quantum version of Maxwell tensor is

$$
4 \pi\left\langle\hat{T}_{i j}\right\rangle_{0}=\left\langle\hat{E}_{i} \hat{E}_{j}\right\rangle_{0}-\frac{1}{2} \delta_{i j}\left\langle\hat{\mathbf{E}}^{2}\right\rangle_{0}+\left\langle\hat{B}_{i} \hat{B}_{j}\right\rangle_{0}-\frac{1}{2} \delta_{i j}\left\langle\hat{\mathbf{B}}^{2}\right\rangle_{0}
$$

Making use of the correlator given by (32) we obtain the following partial results

$$
\begin{gathered}
\left\langle\hat{E}_{x}^{2}(z, t)\right\rangle_{0}=\left\langle\hat{E}_{y}^{2}(z, t\rangle_{0}=\left(\frac{\pi}{a}\right)^{4} \frac{2}{3 \pi}\left[-\frac{1}{120}+F(\xi)\right],\right. \\
\left\langle\hat{E}_{z}^{2}(z, t\rangle_{0}=\left(\frac{\pi}{a}\right)^{4} \frac{2}{3 \pi}\left[\frac{1}{120}+F(\xi)\right],\right.
\end{gathered}
$$

also $\left\langle\hat{E}_{x}(z, t) \hat{E}_{y}(z, t)\right\rangle_{0}=\left\langle\hat{E}_{x}(z, t) \hat{E}_{z}(z, t)\right\rangle_{0}=\left\langle\hat{E}_{y}(z, t) \hat{E}_{z}(z, t)\right\rangle_{0}=0$. In the same way, making use of (35) we obtain

$$
\begin{gathered}
\left\langle\hat{B}_{x}^{2}(z, t)\right\rangle_{0}=\left\langle\hat{B}_{y}^{2}(z, t\rangle_{0}=\left(\frac{\pi}{a}\right)^{4} \frac{2}{3 \pi}\left[-\frac{1}{120}-F(\xi)\right],\right. \\
\left\langle\hat{B}_{z}^{2}(z, t\rangle_{0}=\left(\frac{\pi}{a}\right)^{4} \frac{2}{3 \pi}\left[\frac{1}{120}-F(\xi)\right]\right.
\end{gathered}
$$

and also $\left\langle\hat{B}_{x}(z, t) \hat{B}_{y}(z, t)\right\rangle_{0}=\left\langle\hat{B}_{x}(z, t) \hat{B}_{z}(z, t)\right\rangle_{0}=\left\langle\hat{B}_{y}(z, t) \hat{B}_{z}(z, t)\right\rangle_{0}=0$. The components of the quantum version of Maxwell tensor can be easily evaluated. For instance

$$
\begin{aligned}
8 \pi\left\langle\hat{T}_{z z}(z, t)\right\rangle_{0}= & \left\langle\hat{E}_{z}^{2}(z, t)\right\rangle_{0}-\left\langle\hat{E}_{x}^{2}(z, t)\right\rangle_{0}-\left\langle\hat{E}_{y}^{2}(z, t)\right\rangle_{0} \\
& +\left\langle\hat{B}_{z}^{2}(z, t)\right\rangle_{0}-\left\langle\hat{B}_{x}^{2}(z, t)\right\rangle_{0}-\left\langle\hat{B}_{y}^{2}(z, t)\right\rangle_{0} .
\end{aligned}
$$

performing the necessary substitutions we have

$$
\left\langle\hat{T}_{z z}\right\rangle_{0}=\frac{\pi^{2}}{240 a^{4}}
$$

In the same way

$$
\begin{aligned}
\left\langle\hat{T}_{x x}\right\rangle_{0} & =\left\langle\hat{T}_{y y}\right\rangle_{0}=-\frac{1}{8 \pi}\left(\left\langle\hat{E}_{z}^{2}(z, t)\right\rangle_{0}+\left\langle\hat{B}_{z}^{2}(z, t)\right\rangle_{0}\right) \\
& =-\frac{\pi^{2}}{720 a^{4}}
\end{aligned}
$$

Notice how conveniently the divergent parts near the plates

cancel out yielding finite results. 
Let us obtain now the Casimir force per unit area between the conducting plates. Consider Figure (V) and the plate at $z=a$. The Casimir force per unit area on this plate is

$$
\begin{aligned}
\frac{F_{z}}{A} & =-T_{z z}\left(z \rightarrow a^{L}\right)+T_{z z}\left(z \rightarrow a^{R}\right) \\
& =-\frac{\pi^{2}}{240 a^{4}}+\frac{\pi^{2}}{240(\ell-a)^{4}},
\end{aligned}
$$

where $z \rightarrow a^{L, R}$ means that $z$ tends to $a$ from the left/right. Taking the limit $\ell \rightarrow \infty$ we obtain the expected result for the Casimir force per unit area

$$
\frac{F_{z}}{A}=-\frac{\pi^{2}}{240 a^{4}}
$$

The minus sign shows that the resulting pressure pushes towards the region between the plates. If simultaneously we take the limits $\ell, a \rightarrow \infty$ keeping the distance $\ell-a$ constant. The pressure changes its sign but it still pushes the plate at $z=a$ towards the one at $z=\ell$.

In order to calculate the renormalized symmetrical stressenergy tensor $\left\langle\hat{\Theta}^{\mu \nu}(z)\right\rangle_{0}^{r e n}$ we evaluate the energy density $\rho(z) \equiv\left\langle\hat{\Theta}^{00}(z)\right\rangle_{0}^{r e n}$ in the region between the plates as well as the saptial components $\left\langle\hat{\Theta}^{x x}(z)\right\rangle_{0}^{r e n},\left\langle\hat{\Theta}^{y y}(z)\right\rangle_{0}^{r e n}$, and $\left\langle\hat{\Theta}^{z z}(z)\right\rangle_{0}^{r e n}$. The energy density is given by

$$
\rho(\mathbf{r}, t)=\frac{1}{8 \pi}\left(\left\langle\hat{\mathbf{E}}^{2}(\mathbf{r}, t)\right\rangle_{0}+\left\langle\hat{\mathbf{B}}^{2}(\mathbf{r}, t)\right\rangle_{0}\right)
$$

making use of the correlators given by (32) and (35) we obtain

$$
\rho(a)=-\frac{\pi^{2}}{720 a^{4}} \text {. }
$$

This result is due to the fact that the divergent pieces in (32) and (35) cancel out yielding a finite result for the vacuum energy density. Recalling that $\Theta^{i j}(z)=-T_{i j}(z)$, see [11], with help of (2), (32) and (35) the remanescent components of the symmetrical stress-energy tensor are easily obtained. The final result is

$$
\left\langle\hat{\Theta}^{\mu v}(z)\right\rangle_{0}^{r e n}=\frac{\pi^{2}}{720 a^{4}} \operatorname{diag}(-1,1,1,-3),
$$

which is in perfect agreement with Brown and Maclay's results [6]. Notice also that $\left\langle\hat{\Theta}_{\mu}^{\mu}(z)\right\rangle_{0}^{r e n}=g_{\mu v}\left\langle\hat{\Theta}^{\mu \nu}(z)\right\rangle_{0}^{r e n}=0$, with $g_{\mu v}=\operatorname{diag}(1,-1,-1,-1)$.

\section{THE CASIMIR EFFECT FOR ONE CONDUCTING PLATE AND AN INFINITELY PERMEABLE ONE}

Let us consider now the setup proposed by Boyer [10] which consists of a perfectly conducting plate placed perpendicularly to the $O Z$ axis at $z=0$ and another infinitely permeable one parallel to the first placed at $z=a$. The boundary conditions on the conducting plate are as before $E_{x}=E_{y}=0$ and $B_{z}=0$, and for the infinitely permeable plate: $B_{x}=B_{y}=0$ and $E_{z}=0$. Making use of the correlator given by (55) the following partial results:

$$
\begin{aligned}
\left\langle\hat{E}_{x}^{2}(z, t)\right\rangle_{0} & =\left\langle\hat{E}_{y}^{2}(z, t\rangle_{0}=\left(\frac{\pi}{a}\right)^{4} \frac{2}{3 \pi}\left[\frac{7}{8} \times \frac{1}{120}+G(\xi)\right]\right. \\
\left\langle\hat{E}_{z}^{2}(z, t\rangle_{0}\right. & =\left(\frac{\pi}{a}\right)^{4} \frac{2}{3 \pi}\left[\left(-\frac{7}{8}\right) \times \frac{1}{120}+G(\xi)\right]
\end{aligned}
$$

and $\quad\left\langle\hat{E}_{x}(z, t) \hat{E}_{y}(z, t)\right\rangle_{0}=\left\langle\hat{E}_{x}(z, t) \hat{E}_{z}(z, t)\right\rangle_{0}=$ $\left\langle\hat{E}_{y}(z, t) \hat{E}_{z}(z, t)\right\rangle_{0}=0$. By the same token making use of the correlator given by (56) we obtain

$$
\begin{aligned}
\left\langle\hat{B}_{x}^{2}(z, t)\right\rangle_{0} & =\left\langle\hat{B}_{y}^{2}(z, t\rangle_{0}=\left(\frac{\pi}{a}\right)^{4} \frac{2}{3 \pi}\left[\frac{7}{8} \times \frac{1}{120}-G(\xi)\right]\right. \\
\left\langle\hat{B}_{z}^{2}(z, t\rangle_{0}\right. & =\left(\frac{\pi}{a}\right)^{4} \frac{2}{3 \pi}\left[\left(-\frac{7}{8}\right) \times \frac{1}{120}-G(\xi)\right]
\end{aligned}
$$

and also

$$
\left\langle\hat{B}_{x}(z, t) \hat{B}_{y}(z, t)\right\rangle_{0}=\left\langle\hat{B}_{x}(z, t) \hat{B}_{z}(z, t)\right\rangle_{0}=\left\langle\hat{B}_{y}(z, t) \hat{B}_{z}(z, t)\right\rangle_{0}=0
$$

Proceeding as in the case of the conducting plates we obtain the following results for the componenets of the quantum version of Maxwell tensor

$$
\left\langle\hat{T}_{x x}\right\rangle_{0}=\left\langle\hat{T}_{y y}\right\rangle_{0}=\frac{7}{8} \times \frac{\pi^{2}}{720 a^{4}},
$$

and

$$
\left\langle\hat{T}_{z z}\right\rangle_{0}=\left(-\frac{7}{8}\right) \times \frac{\pi^{2}}{240 a^{4}} .
$$

Notice that it is sufficient to multiply the results obtained for Casimir's setup by the factor $(-7 / 8)$ in order to obtain the results corresponding to Boyer's setup.

In order to obtain the Casimir force per unit area for this setup it is convenient to place a third conducting plate at $z=\ell$. Then the Casimir force per unit area on the plate at $z=a$ will be given by

$$
\begin{aligned}
\frac{F_{z}}{A} & =-T_{z z}\left(z \rightarrow a^{L}\right)+T_{z z}\left(z \rightarrow a^{R}\right) \\
& =-\left(-\frac{7}{8}\right) \times \frac{\pi^{2}}{240 a^{4}}+\left(-\frac{7}{8}\right) \times \frac{\pi^{2}}{240(\ell-a)^{4}}
\end{aligned}
$$

Taking the limit $\ell \rightarrow \infty$ we obtain a Casimir force which pushes the plate at $z=a$ towards the region $z>a$ given by

$$
\frac{F_{z}}{A}=\frac{7}{8} \times \frac{\pi^{2}}{240 a^{4}}
$$

This is the result obtained by Boyer [10] for this setup using stochastic electrodynamic methods and it is one of the simplest example of a repulsive Casimir force. 
In order to evaluate the symmetrical stress-energy tensor we first evaluate the Casimir energy density. Making use of (55) e (56) we have

$$
\rho=\frac{7}{8} \times \frac{\pi^{2}}{240 a^{4}}
$$

As in the case of the conducting plates a simple calculation shows that the stress energy tensor for Boyer's setup is given by

$$
\left\langle\hat{\Theta}^{\mu \nu}(z)\right\rangle_{0}^{r e n}=\frac{7}{8} \times \frac{\pi^{2}}{720 a^{4}} \operatorname{diag}(1,-1,-1,3) .
$$

As before $\left\langle\hat{\Theta}_{\mu}^{\mu}(z)\right\rangle_{0}^{r e n}=g_{\mu \nu}\left\langle\hat{\Theta}^{\mu \nu}(z)\right\rangle_{0}^{r e n}=0$.

\section{CONCLUSIONS}

In this paper we have shown how to employ the equal time and space electromagnetic field correlators evaluated between parallel material surfaces to rederive results concernig the Casimir energy and pressure and the symmetrical traceless stress energy tensor. We have shown that for the cases we had in mind here finite results are obtained only when we consider what happens on both sides of the surface boundary. This consideration provided the mechanism by which precise cancellations occurred and finite results were obtained. These cancellations occur only for the simple geometry considered in this paper. This is in agreement with, for example, Ref. [9] and should be considered as a concrete example of the behavior of quantized fields near and on boundary surfaces. This behavior depends on the geometry of the boundaries and can become quite complicated. It can depend on the local curvature of the boundary, for instance. Finally, it must be mentioned that the equal time and space electromagnetic field correlators calculated here were can be also employed to rederive the atractive and the repulsive Casimir effect by means of a quantum version of the Lorentz force [20]. They can be also applied to the Casimir-Polder interaction between an atom and material surfaces [21]
[1] H. B. G. Casimir, Proc. K. Ned. Akad. Wet. 51, 793 (1948); Philips Res. Rep. 6. 162 (1951).

[2] M. J. Sparnaay, Physica 24, 751 (1958).

[3] S. K. Lamoreaux, Phys. Rev. Lett. 78, 5 (1997); erratum Phys. Rev. Lett. 81, 5475 (1988); U. Mohideen and A. Roy, Phys. Rev. Lett. 81, 4549 (1998).

[4] M. Bordag, U. Mohideen and V.M. Mostepanenko, Phys. Rep. 353, 1 (2001); quant-ph/0106045.

[5] V. M. Mostepanenko and N. N. Trunov, Sov. Phys. Usp. 31, 965 (1988); V. M. Mostepanenko and N. N. Trunov, The Casimir Effect and its Applications, (Oxford, Clarendon, 1997); G. Plunien, B. Müller and W. Greiner, Phys. Rep. 134, 664 (1987); S. K. Lamoreaux, Am. J. Phys. 67, 850 (1999). See also: M.V. Cougo-Pinto, C. Farina and A. C. Tort, Rev. Bras. Ens. Fís. 22 122 (2000) (in Portuguese).

[6] L.S. Brown and G.J. Maclay, Phys. Rev. 184, 1272 (1969).

[7] A. E. Gonzales, Physica 131A 228-236 (1985).

[8] K. A. Milton,The Casimir Effect: Physical Manifestations of Zero-Point Energy (World Scientific, Singapore, 2001).

[9] D. Deutsch and P. Candelas, Phys. Rev. D 20, 3063 (1979).

[10] T.H. Boyer Phys. Rev. A 9, 2078 (1974).

[11] J. D. Jackson, Classical Electrodynamics, 3rd. ed., (John Wiley, New York 1999).
[12] G. Barton, Phys. Lett. B 237, 559 (1990).

[13] M. V. Cougo-Pinto, C. Farina , F. C. Santos and A. C. Tort: J. of Phys. A 32 (1999), 4463.

[14] H.B.G. Casimir, J. Chim. Phys. 46, 407 (1948).

[15] T.H. Boyer, Phys. Rev. 180, 19 (1969).

[16] C.A. Lütken and F. Ravndal, Phys. Rev. A 31, 2082 (1985).

[17] M. Bordag, D. Robaschik and E. Wieczorek, Ann. Phys. (NY) 165, 192 (1985).

[18] E. Elizalde, S. D. Odintsov, A. Romeo, A. A. Bytsenko and S. Zerbini: Zeta Regularization Techniques with Applications, World Scientific, Singapore (1994). See also R. Ruggiero, A. H. Zimerman and A. Villani, Rev. Bras. Fís. 7, 663 (1977). For a simple introduction to the Zeta function regularization technique see: J. J. Passos Sobrinho and A. C. Tort, Rev. Bras. Ens. Fís. 23, 401 (2001) (in Portuguese).

[19] I. S. Gradshteyn and I. M. Ryzhik, Tables of Integrals, Series and Products, 5th Edition, Academic Press, New York (1994).

[20] C. Farina, F. C. Santos and A. C. Tort, Eur. J. of Phys. 24 N5-N9 (2003).

[21] F. C. Santos, J. J. Passos Sobrinho, and A. C. Tort, Braz. J. of Phys. 35136 (2005). 